\title{
Karst Rocky Desertification analysis based on historical DISP data take two regions for example
}

\author{
Jie Liu ${ }^{1,2, *}$, Guoqing Zhou ${ }^{1,2}$, Bin Jia ${ }^{1,2, *}$, Tao Yue ${ }^{1}$, Xinyue Peng ${ }^{1}$ \\ ${ }^{1}$ Guangxi Key Laboratory of Spatial Information and Geomatics, Guilin University of Technology, No. 12 Jian'gan Road, Guilin, \\ Guangxi, 1004, China - gzhou@glut.edu.cn \\ ${ }^{2}$ College of Earth Sciences, Guilin University of Technology, No. 12 Jian'gan Road, Guilin, Guangxi,
} 541004, China - jiel36@foxmail.com

KEY WORDS: DISP Imagery, Karst Rocky Desertification, Visual Interpretation, Georeferencing, Raster map, Vectorization

\begin{abstract}
:
Karst rocky desertification (KRD) is used to characterize the processes that transform a karst area covered by vegetation and soil into a rocky landscape almost devoid of soil and vegetation. This situation seriously affects and threatens the living environment and standards of local people, which results in a series of social problems. In view of the importance and harmfulness of KRD, many scholars have studied the spatial and temporal evolution of KRD and its driving forces. In this paper, the Visual Interpretation Marks of Rocky Desertification in Southwest China in 1960s are constructed by using the DISP image of the United States, combined with DEM data and Hydrogeological data. The area of rocky desertification in Guangnan and Funing counties, where rocky desertification is more serious, is about $2457.729 \mathrm{Km}^{2}$. The area of rocky desertification can be used as the basic data for studying the historical changes in southwestern China by researchers.
\end{abstract}

\section{INTRODUCTION}

Karst rocky desertification (KRD) is used to characterize the processes that transform a karst area covered by vegetation and soil into a rocky landscape almost devoid of soil and vegetation (Yuan, 1997; Wang et al., 2004a). Research has shown that $\mathrm{KRD}$ is one main type of land desertification which is caused by irrational human impact on the vulnerable eco-geo-environment (Wang S et al., 2004). Karst regions in Southwest China are some of the largest exposed carbonate rock areas in the world(Jiang et al., 2014), Guangnan and Funing counties are the more serious KRD areas in Southwest China. KRD has attracted more and more attention from all over the world. Therefore, The long time series of KRD data can help to understand the impact of rocky desertification on natural and human activities and provide insights into the protection and sustainable development of karst areas without rocky desertification. In order to study karst rocky desertification in Guangnan and Funing counties using DISP images in 1960s, it is necessary to preprocess DISP images, but there are still some unknown imaging parameters in CORONA satellite. As a result, In this paper, the polynomial model inserting elevation correction term is used for geometric correction of 16 image data, then the radiating treatment and seamless splicing are carried out, obtain KH-4B High Resolution Panchromatic Images in Guangnan and Funing counties. After downloaded ASTER GDEM2 data are spliced to obtain the slope map. After that, splice the downloaded ASTER GDEM2 data and obtain the slope map. Using slope map, Hydrogeological data and land use map as auxiliary data, combine with KH-4B Image and Documentation Confirmation identifying visual interpretation signs, using Arcgis and Envi software to interpret KH-4B image by human-computer interaction, and finally the rocky desertification area of Guangnan and Funing counties was about $2457.729 \mathrm{Km}^{2}$.

\section{RESEARCH AREAS AND DATA SET}

\subsection{Research areas}

The study areas are Guangnan and Funing counties. The reason for choosing these two areas is that the two areas are located in Yunnan Province where Karst Rocky Desertification is more serious in southwestern China (Figure 1). Guangnan and Funing counties ranging from $104^{\circ} 31^{\prime}$ to $1106^{\circ} 12^{\prime} \mathrm{E}$ and from $23^{\circ} 11^{\prime}$ to $24^{\circ} 28^{\prime} \mathrm{N}$, its land area is $13,162 \mathrm{Km}^{2}$. Guangnan and Funing counties has a humid monsoon climate of north subtropics with an average annual temperature of $16.7^{\circ} \mathrm{C}$. Total annual precipitation in the area is about $1080 \mathrm{~mm}$, wet-season which comes between May and October, and terrain in this area has an elevation between $420 \mathrm{~m}$ and $2035 \mathrm{~m}$ above sea level (http://www.yngn.gov.cn and http://www.ynfn.gov.cn).

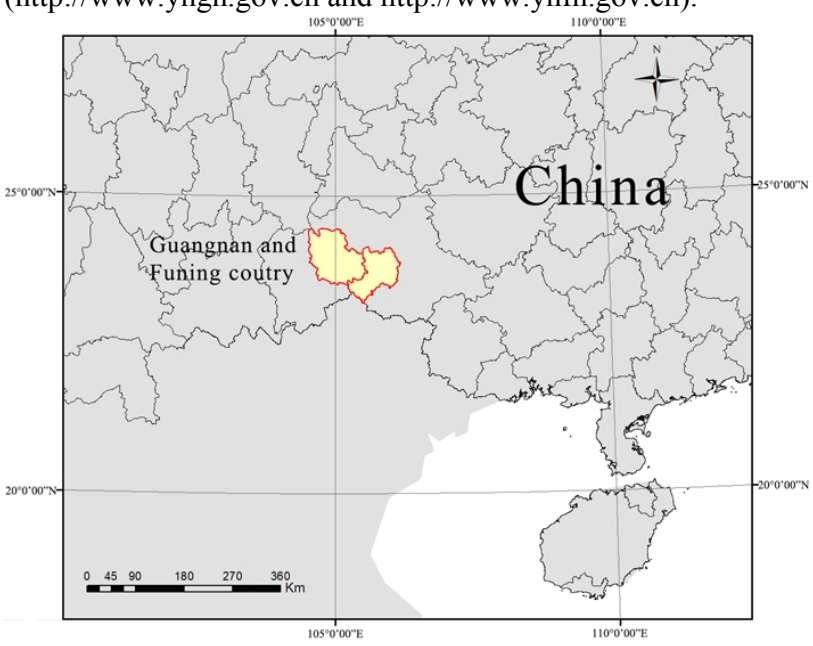

Figure 1. Location map of Guangnan and Funing counties,

\section{China}

Guangnan and Funing counties are located in the second step area of China's geomorphic unit, and the absolute elevation gradually rises to the West as a whole (Figure 2). The

\footnotetext{
* Corresponding author
} 
geomorphologic types are mainly Peak Cluster Depression, and the slope is generally more than 20 . Marine carbonate rocks (dolomites) dominate the Sinian; The Cambrian is dominated by marine carbonate (dolomite) interbedded with terrestrial clastic rocks; Apart from more clastic rocks in Lower Ordovician, almost all of Middle-Upper Ordovician are limestone; Permian carbonate rocks are mostly pure limestone; The Triassic is dolomite and limestone deposits. The proportion of dolomite increases gradually and then transits to clastic rocks (Bureau., 1990).

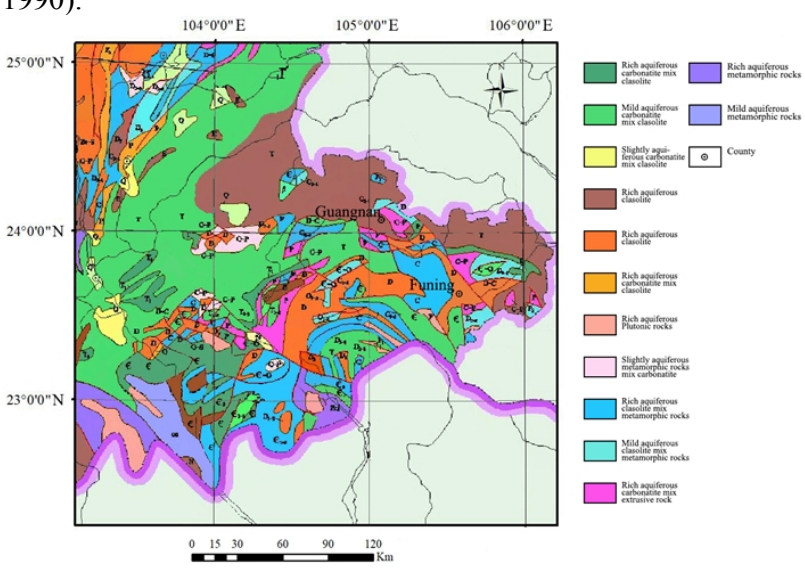

Figure 2. Hydrogeological Map

\subsection{DATA SET}

From 1959 to the 1980 s, the U.S. launched several reconnaissance satellite programs that acquired a large number of images (United States Geological Survey, 2015). On February 22, 1995, President William j. Clinton (1995) signed Executive Order 12951, which declas-sified Satellite Imagery from the first generation of U.S. reconnaissance satellites, known as DISO-1. In this study, we used KH-4B from DISP-1. Also Data sources involved in the Karst Rocky Desertification Visual interpretation Aster Gdem2 are from the USGS. The Hydrogeological data are derived from the karst scientific data center. In addition, Land utilization map, local administrative maps, information from previous research and the yearbooks of Guangnan and Funing counties are used as supporting data in the study (Table 1).

Table 1. The main data source

\begin{tabular}{ccc}
\hline Data Name & Data Source & Website Link \\
\hline DISP KH-4B & USGS & https://earthexplorer.usgs.gov/ \\
\hline Aster Gdem2 Data & USGS & https://earthexplorer.usgs.gov/ \\
\hline Hydrogeological Data & $\begin{array}{c}\text { Karst Science } \\
\text { Data Center }\end{array}$ & http://www.karstdata.cn/ \\
\hline $\begin{array}{c}\text { Local administrative } \\
\text { Maps }\end{array}$ & $\begin{array}{c}\text { GADM maps and } \\
\text { data }\end{array}$ & http://www.gadm.org \\
\hline Land utilization Map & $\begin{array}{c}\text { Yunnan } \\
\text { geological survey }\end{array}$ & http://ddj.yndlr.gov.cn/ \\
\hline & &
\end{tabular}

\section{KARST ROCKY DESERTIFICATION RESEARCH METHODS}

\subsection{Karst Carbon Sink Research Methods}

The proposed approach consists of three main steps: (1) The quadratic polynomial model is used to correct the KH-4B image without any satellite parameters, at the same time, in order to eliminate the error caused by terrain undulation, the elevation difference correction is added into the model, 12 images were mosaic and radiation balance processing to obtain $\mathrm{KH}-4 \mathrm{~B}$ images of Guangnan and Funing counties in the 1960s. (2) The slope maps of Guangnan and Funing counties were made by using the Surface Fitting Method (Arthur N Strahler. 1956) for Aster Gdem2 Data. (3) Visual Interpretation of Guangnan and Funing counties combine with Hydrogeological Data and Slope Data. Each step is described in detail in the following sections.

\subsection{Image preprocessing}

Because the parameters of the satellite are unknown, such as the inner and Outer Orientation Elements and the Camera Lens Distortion Parameters. In this study, polynomial model is used to correct image. In the process of correction, the elevation term is added, and a three-dimensional quadratic polynomial model is established to eliminate the deformation caused by the elevation difference term. The model is mainly applied to small images or images with systematic geometric correction. S. Vassilopoulou combines DEM with quadratic polynomial model for ortho-rectification of IKONOS images, the result of correction is much better than that of two-dimensional quadratic polynomial model without elevation term, especially in mountainous areas (Vassilopoulou S et al., 2004). The process of establishing the model is as follows:

Two-dimensional polynomial extended to three-dimensional polynomial model:

$$
\left\{\begin{array}{c}
X_{T}=\sum_{i=0}^{M} \sum_{j=0}^{N} \sum_{k=0}^{o} a_{i j} \cdot X_{D}^{i} Y_{D}^{j} Z^{k} \\
Y_{T}=\sum_{i=0}^{M} \sum_{j=0}^{N} \sum_{k=0}^{O} b_{i j} \cdot X_{D}^{i} Y_{D}^{j} Z^{k}
\end{array}\right\}
$$

The expansion of the three-dimensional polynomial model is as follows:

$$
\left\{\begin{array}{l}
X_{T}=a_{0}+a_{1} X_{D}+a_{2} Y_{D}+a_{3} Z+\ldots+a_{7} Z+a_{8} X_{D} Z+a_{9} Y_{D} Z+\ldots \\
Y_{T}=b_{0}+b_{1} X_{D}+b_{2} Y_{D}+b_{3} Z+\ldots+b_{7} Z+b_{8} X_{D} Z+b_{9} Y_{D} Z+\ldots
\end{array}\right\}
$$

Compared with the binomial model, the three-dimensional polynomial model only adds more Z-elevation terms than the two-dimensional polynomial model, so the solving process is the same. Adding elevation item means adding elevation correction, So the three-dimensional polynomial model can be said to be ortho-rectification model (Huang et al., 2008).

The model expression is:

$$
\left\{\begin{array}{l}
X_{T}=a_{0}+a_{1} X_{D}+a_{2} Y_{D}+a_{3} X_{D}^{2}+a_{4} X_{D} Y_{D}+a_{5} Y_{D}^{2}-(H-Z) Z / R / S \\
Y_{T}=b_{0}+b_{1} X_{D}+b_{2} Y_{D}+b_{3} X_{D}^{2}+b_{4} X_{D} Y_{D}+b_{5} Y_{D}^{2}
\end{array}\right\}
$$

Where $R$ is the slope distance, $S$ is the dimension of distance oriented pixel, $H$ is the Row height.

The formula of number of points selected according to the polynomial correction model:

$$
N=(n+1)(n+2) / 2
$$

Where $N$ is the minimum number of homonyms selected, $n$ is the Subclasses in Polynomials.

When correcting, The linear polynomial correction modeler must select at least three control points in order to obtain the coefficients in the model, quadratic polynomials require at least six control points, cubic polynomials require at least 10 control points (Toutin T et al., 2004). Because the image that needs to be corrected has been more than 50 years ago, it is difficult to find the image or map of the same period as reference data, so there are some problems in finding and selecting control points. 
This study chooses the image of Google Earth as reference data to correct the image of the 1960s.

$\mathrm{KH}-4 \mathrm{~B}$ image has a large coverage area of $16 \times 217 \mathrm{Km}$. In order to ensure the accuracy of correction, 120 control points are selected for each image to correct the image. In order to improve the accuracy of point selection, this paper chooses control points on obvious features such as road inflection points, bridges, surrounding buildings, River inflection points and intersection points. After geometric correction of each scene image, Envi Seamless mosaic (ESRI, Redlands, CA, USA) is used for stitching, histogram equalization (citation) is used to complete radiation balance processing, and SHP of Guangnan and Funing counties Administrative Region is used to clip the image. Finally, KH-4B images of Guangnan and Funing counties in the 1960 s are obtained (Figure 3).

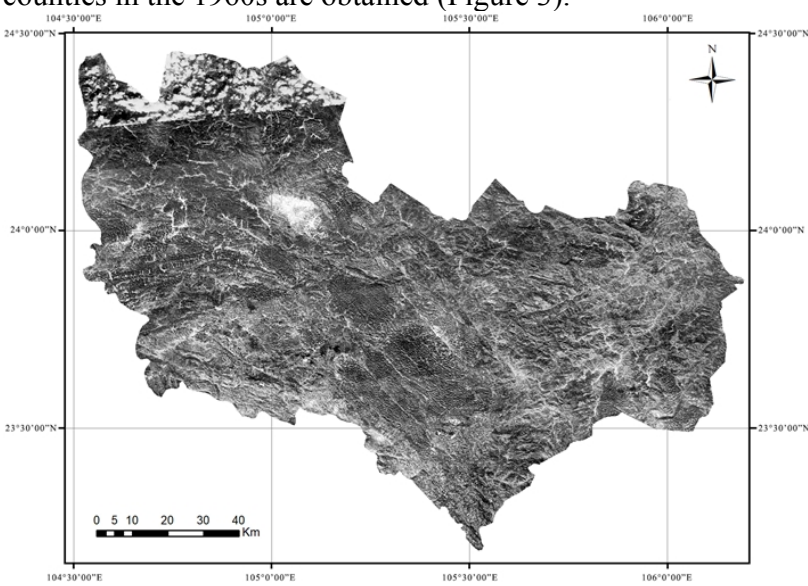

Figure 3. KH-4B images of Guangnan and Funing counties in the $1960 \mathrm{~s}$

\subsection{Make Slope map}

Slope is one of the main factors resulting in karst rocky desertification. In Karst mountain area southwest China, areas with slope $>15^{\circ}$ are not suitable for farming because it would accelerate soil erosion, which would change the farmland into barren soil. The central government also promulgate decrees that farmland with slope $>25^{\circ}$ must be changed into forest or grassland to protect topsoil, and areas with the slope $<5^{\circ}$ is nearly suitable for all kinds of land use. All disciplines of scientific research involving studies of the earth's land surface require topographic data such as elevation, slope and aspect (Topographic Science Working Group, 1988). Slope is among the most important data in many natural resource spatial databases. (Bolstad and Stowe, 1994). In this paper, the slope value is calculated by fitting a pixel with the $Z$ value of a $3 \times 3$ neighborhood around the pixel to be processed or the central pixel, and then calculating the maximum average value (Burrough and McDonell, 1998).

Slope formula:

$$
\begin{aligned}
& \text { Slope }=\tan \sqrt{S_{x}^{2}+S_{y}^{2}} \\
& S_{x}=\frac{e_{1}-e_{3}}{2 \times \text { Cellsize }} \\
& S_{y}=\frac{e_{4}-e_{2}}{2 \times \text { Cellsize }}
\end{aligned}
$$

Where Slope is slope value, $S_{x}$ is Slope in $x$ direction, $S_{y}$ is Slope in $y$ direction.

After calculating the slope of each scene with Aster Gdem2, Envi Seamless mosaic is used for stitching, and then SHP of Guangnan and Funing counties Administrative Region is used to cut the image. Finally, the slope maps of Guangnan and Funing counties in the 1960 s are obtained (Figure 4).

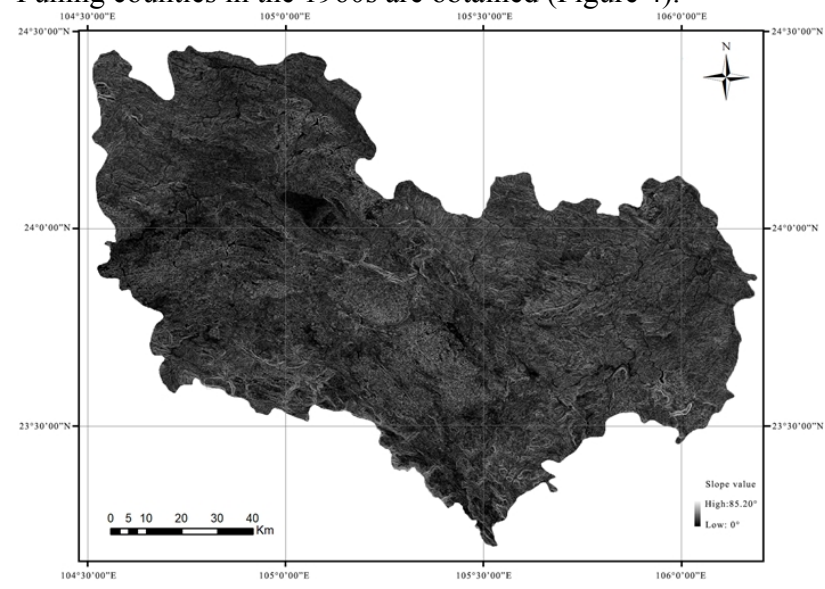

Figure 4. Slope map of Guangnan and Funing counties

\subsection{Visual interpretation}

Because KH-4B image is a high-resolution single-band panchromatic image with little spectrum information, it is difficult to extract KDR information by traditional and deep learning methods or models. Therefore, combined with DEM, slope data, hydrogeologic data, land use data, previous research data and Guangnan and Funing Yearbook as auxiliary data, the visual interpretation of rocky desertification is carried out by human-computer interaction through ArcGIS 10.5 software (ESRI, Redlands, CA, USA). Visual interpretation should first establish interpretation signs. There are four factors influencing the manifestation of KRD on KH-4B images: Hue, Form, Position, Tense (Table 2).

Table 2 Visual interpretation marks

\begin{tabular}{c|l|l|l}
\hline $\begin{array}{c}\text { Influence } \\
\text { factor }\end{array}$ & \multicolumn{1}{|c|}{ Embody } & \multicolumn{1}{c}{ Effect } & \multicolumn{1}{c}{ KRD } \\
\hline Hue & $\begin{array}{l}\text { Radiation } \\
\text { Brightness Value }\end{array}$ & $\begin{array}{l}\text { Distinguish target } \\
\text { attributes }\end{array}$ & $\begin{array}{l}\text { Shallow hue: Marble, } \\
\text { Quartzite } \\
\text { Middle hue: Limestone, } \\
\text { Dolomite } \\
\text { Dark hue: Soil Layer Rich } \\
\text { in Organic Matter and } \\
\text { Fonse vegetation }\end{array}$ \\
\hline Position & $\begin{array}{l}\text { Shape、Size、T } \\
\text { exture }\end{array}$ & $\begin{array}{l}\text { Distinguish target } \\
\text { attributes and area }\end{array}$ & $\begin{array}{l}\text { Planar or linear } \\
\text { Orange peel-like texture }\end{array}$ \\
\hline Tense & nt Relations & $\begin{array}{l}\text { Distinguish target } \\
\text { attributes and } \\
\text { boundary }\end{array}$ & $\begin{array}{l}\text { Controlled by geological } \\
\text { structures } \\
\text { Strip, Round, Chessboard }\end{array}$ \\
\hline
\end{tabular}

One of the necessary conditions for the occurrence of karst rocky desertification is that the lithology with a certain thickness exposed to the surface is carbonate or silicate rock. According to the Hydrogeological map, the non-karst areas can be excluded, and then the land use data can be used to exclude the man-made construction of cities and towns, roads and so on. Combining with the overlapping areas of areas with slope ( $>20$ degrees) and visual interpretation of KH-4B images, the karst rocky desertification area of Guangnan and Funing counties in the $1960 \mathrm{~s}$ is about $2457.729 \mathrm{Km}^{2}$. This comprehensive information interpretation method using spatial reasoning and geological elements can quickly and accurately depict rocky desertification areas (Figure 5) and the red region is KDR. 


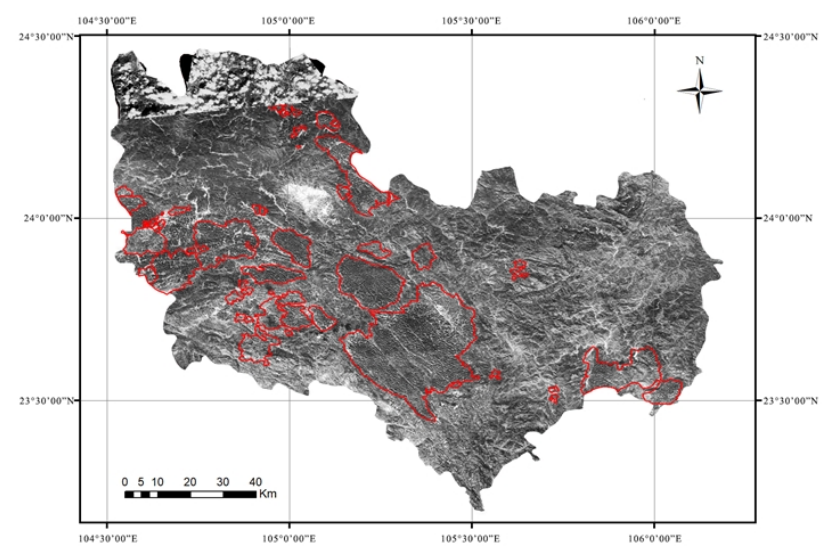

Figure 5. KH-4B images of Guangnan and Funing counties

KRD in the 1960 s

\subsection{Accuracy assessment}

We use Envi to select 20 control points randomly to project the corrected KH-4B image onto the Google image, and calculate the distance between the projection point and the real point in the horizontal and vertical directions. After validating the corrected image, it is found that the corrected KH-4B image has errors of 1 to 3 pixels in both $\mathrm{X}$ and $\mathrm{Y}$ directions. The vertical and horizontal distance errors are about 2 to 3 meters and 2 to 7 meters respectively.

\section{CONCLUNSIONS}

During the great leap forward period, due to large-scale smelting of steel, urban construction and the population increased on a large scale. In order to human meet the minimum survival needs, a large number of trees were cut down, which led to a vicious circle of reclamation with more poverty.

However, in the 1960s were no statistics on karst rock desertification, and only a brief karst rock desertification description in the general history of a county. This work constructed visual interpretation marks of KRD in southwestern China from DISP images in the 60's, offers 60's Guangnan and Funing counties karst rock desertification area about $2457.729 \mathrm{Km}^{2}$ and providing a basis for the study of karst rock desertification historical changes in southwest China.

\section{ACKNOWLEDGEMENTS}

This paper is financially supported by the National Natural Science of China under Grant numbers 41431179,41961065; Guangxi Innovative Development Grand Grant under the grant numbers GuikeAA18118038, GuikeAA18242048; the National Key Research and Development Program of China under Grant number 2016YFB0502501 and the BaGuiScholars program of Guangxi (Guoqing Zhou).

\section{REFERENCES}

DX, Yuan, 1997. Rock desertification in the subtropical Karst of South China. Zeitschrift fur Geomorphologie, Supplementband. vol. 108, pp. 81-90.

Wang, S. J., Li, R. L., Sun, C. X., Zhang, D. F., Li, F. Q., Zhou, D.Q., Xiong, K. N., and Zhou, Z. F., 2004. How types of carbonate rock assemblages constrain the distribution of karst rocky desertified land in Guizhou Province, PR China: phenomena and mechanisms, Land Degrad. Dev., vol. 15, 123 131 ,
Wang S, Liu Q., 2004. Karst rocky desertification in southwestern China: geomorphology, land use, impact and rehabilitation. Land Degrada Develop, vol. 15, pp.115-121.

Jiang, Z.C., Lian, Y.Q., Qin, X.Q., 2014. Rocky desertification in Southwest China: Impacts, causes, and restoration. Earth Sci. Rev,vol. 132, pp.1-12.

Bureau of Geology and Mineral Resources of Yunnan Province., 1990. Regional Geology of Yunan Province. Geological Publishing House, Beijing, pp. 728. (in Chinese with English brief text).

United States Geological Survey., 2015. Website: in: http://glovis.usgs.gov/

Arthur N Strahler., 1956. Quantitative Slope Analysis. GSA Bulletin, vol. 67, no.5, pp. 571-596.

Vassilopoulou S, Hurni L, Dietrich V, et al., 2002. Orthophoto generation using IKONOS imagery and high-resolution DEM: A case study on volcanic hazard monitoring of Nisyros Island (Greece). ISPRS Journal of Photogrammetry and Remote Sensing, vol. 57, pp. 24-38.

Guoman Huang, Xijuan Yue, Hongdong Fan., 2008. Block Adjustment with Airborne SAR Images Based on Polynomial Ortho-Rectification. Geomatics and Information Science of Wuhan University, vol. 06, pp. 569-572.

Toutin T., 2004. Review article: Geometric processing of remote sensing images: models algorithms and methods [J. International Journal of Remote Sensing, vol.25, no.10, pp. 1893-1924.

Topographic Science Working Group., 1988. Topographic Science Working Group report to the Land Processes Branch. Earth Science and Applications Division, NASA Headquarters: Lunar and Planetary Institute, Houston, TX, pp.64.

Bolstad, P.V., Stowe, T., 1994. An evaluation of DEM accuracy: elevation, slope, and aspect. Photogrammetric Engineering and Remote Sensing, vol. 60, no. 11, pp. $1327-1332$

Burrough, P. A., and McDonell, R. A., 1998. Principles of Geographical Information Systems (Oxford University Press, New York), pp. 190. 